\title{
Feature Generation for Seismic and Acoustic Signals Based on Hilbert-Huang Transform
}

\author{
Roman Berešík, Jozef Puttera and Jozef Jurčo \\ Department of Electronics \\ Armed Forces Academy of Gen. M. R. Štefánik \\ Liptovský Mikuláš, Slovakia \\ roman.beresik@aos.sk,jozef.puttera@aos.sk,jurci0@yahoo.com
}

\begin{abstract}
The successful detection and classification of events require using signal processing algorithms suitable for analysis of nonlinear and nonstationary signals like seismic and acoustic ones. A key point of the signal classification is to generate the feature vector by the means whose signals can be characterized and differentiated from another classes of signals. Some characteristics of seismic and acoustic signals can be described in the frequency and time-frequency domain by using adaptive signal processing methods like the Hilbert-Huang transform. The paper presents results of seismic and acoustic signal processing in the process of feature vector generation by detection of significant frequency components in the energy density spectrum of Hilbert-Huang transform.
\end{abstract}

Keywords-feature generation; feature vector; HilbertHuang transform; seismic and acoustic signals

\section{INTRODUCTION}

A detection and subsequent classification of events by using multisensor systems requires utilization of robust signal processing methods. Subsequently, characteristic features of signals can be successfully identified in the time, frequency and time-frequency domain in order to minimize a false alarm rate. The successful feature generation, as a part of the classification process, requires preprocessing of signals in order to decrease influence of undesired signal components which are not relevant to a particular event and carry out a reduction of signal dimension. Typical applications where the signal feature generation is required are military and security systems used for detection of events like moving wheeled or traced vehicles. Each event has its own unique characteristics, which served as target descriptors for following classification process. One of the most common methods used for feature generation in the frequency domain is discrete Fourier transform (DFT). The DFT implements the law of decomposition and superposition using harmonic sine and cosine functions $[1,2]$. It calculates the Fourier transform (FT) on block by block basis. The size of the block determines the tradeoff between the resolution in time and frequency domain [3]. There are other signal processing methods widely used for seismic and acoustic signal analysis like Wavelet transform (WT) and Wigner-Ville distribution (WVD) [4]. These signal processing methods also have limitations in terms of the time-frequency resolution like DFT has. Characteristics of seismic and acoustic signals, generated by vehicles or another sources define that the most of them have a nonlinear and nonstationary character. One of the methods used for signal analysis of nonlinear and nonstationary seismic signals is Hilbert-Huang transform (HHT) $[4,5]$. The HHT consists of two parts: Empirical Mode Decomposition (EMD) and Hilbert spectral analysis (HSA) [4]. There are several modifications of EMD like the Ensemble Empirical Mode Decomposition (EEMD) proposed in [6] and its modification named Complete Empirical Mode Decomposition (CEEMD) method introduced in [7]. The feature generation is a process of signal transform which represents the particular event as a new set of features with reduced data space. Features as results of the feature generation can be described by structural way, in time domain, like number of local maxima, minima, relative time of maxima etc. Another way how to describe features of events is to exploit specific parameters from the frequency or time-frequency domain.

The presented paper is devoted to problems of signal processing of seismic and acoustic signals generated by moving vehicles in terms of the feature generation base on HHT by getting the energy spectra and defining significant frequency components which describe acoustic and seismic signatures of moving vehicles.

\section{PROBLEM DESCRIPTION OF HILBERT-HUANG TRANSFORM}

\section{A. Empirical mode decomposition}

The EMD decomposes an input signal into a certain number of Intrinsic Mode Functions (IMFs). The input signal must follow two conditions [6]:

- The number of extreme and number of zero crossings must be equal or differ at most by one.

- At any point, the mean value of envelope defined by local maxima and envelope defined by local minima is zero.

Measured data sets of seismic and acoustic signals consist of not only a desired signal, describing the event in monitored area, but also a noise other than noise generated by vehicles. The signal can be described as follows

$$
x[t]=s[t]+n[t],
$$

where $\mathrm{s}[t]$ represents the seismic or acoustic event and $n[t]$ represents the noise and interference component of signals. 
The result of the EMD is represented as a set of IMFs, which separate high and low frequency components. The EMD acts as dyadic filter banks where particular IMFs can have variable amplitude and frequency. After the EMD, every IMF is processed by the HSA as a tool for time-frequency representation of analyzed signals.

\section{B. Hilbert Spectral Analysis}

One method how to express nonlinear and nonstationary character of data is by the instantaneous frequency and instantaneous amplitude. The Hilbert transform (HT) of signal $x(\mathrm{t})$ is given by following equation [8].

$$
y(t)=\frac{1}{\pi} P \int_{-\infty}^{\infty} \frac{x(\tau)}{t-\tau} d \tau
$$

where $P$ is Cauchy principal value of the singular integral. With the HT of signal $x(\mathrm{t})$, the analytical signal can be defined as

$$
z(t)=x(t)+i y(t)=a(t) e^{i \theta(t)}
$$

where $i=(-1)^{1 / 2}$.

Subsequently, the $a(\mathrm{t})$ can be expressed as

$$
a(t)=\sqrt{\left(x^{2}+y^{2}\right)},
$$

where $a(t)$ is the instantaneous amplitude. The instantaneous phase function can be expressed as follows

$$
\theta(t)=\arctan \frac{y}{x} .
$$

The instantaneous frequency is given by

$$
\omega=\frac{d \theta}{d t}
$$

Applying the HT to single components of IMFs, the original data can be expressed as a real part of the following equation [4]

$$
x(t)=\operatorname{Re}\left\{\sum_{j=1}^{n} a_{j}(t) \exp \left[i \int \omega_{j}(t) d t\right]\right\} .
$$

The equation (7) expresses the real part of instantaneous amplitude (4) and instantaneous frequency (6) of each IMF component as a function of time. The representation of signal in time-frequency domain can be expressed by Hilbert energy spectrum (HES) or Hilbert amplitude spectrum (HAS). The HES is defined as an energy density distribution and similarly, the HAS is defined as the amplitude density distribution in a time-frequency space [9]. The Hilbert energy density spectrum (HEDS) is defined as

$$
S_{i, j}=H\left(t_{i}, \omega_{j}\right)=\frac{1}{\Delta t \times \Delta \omega} H\left[\sum_{k=1}^{n} a_{k}^{2}(t)\right] .
$$

The resolution of the Hilbert spectrum is given by equal-sized bins expressed as $\Delta t \times \Delta \omega$. Each bin represents the value of $a^{2}(t)$ in given time and frequency. The size of bins could be selected independently and arbitrarily to fit one's needs.

\section{SiesmiC AND ACOUSTIC Signal Processing FOR FEATURE GENERATION}

The seismic-acoustic sensor subsystem used for a measurement contains the micro-electromechanical system (MEMS) seismic accelerometer SF1500S.A [10] and the MEMS microphone ADMP404 [11]. The data were sampled with the data rate equal to $f_{s}=5$ kSPS and 16 bit of resolution. The flowchart of signal processing algorithms is shown in Fig. 1. Due to the fact that measured acoustic and seismic signals comprise the DC offset, which does not carry any information about the event, this signal component was removed before further signal processing. The process of DC removing is described by following equation

$$
x_{\text {in_dc_off }}[n]=x_{\text {in }}[n]-\frac{1}{Z} \sum_{n=1}^{Z} x_{i n}[n],
$$

where $x_{\text {in_ } d c_{-} \text {off }}[n]$ represents the time series of input signals without DC offset, $x_{i n}[n]$ is input time series and $Z$ refers to the number of samples used for computing of DC offset mean. For this particular time series, $Z$ consists of 5,000 samples from the time interval with no seismic or acoustic events. Analyzed acoustic and seismic input signals consist of $n=32,000$ samples of data.

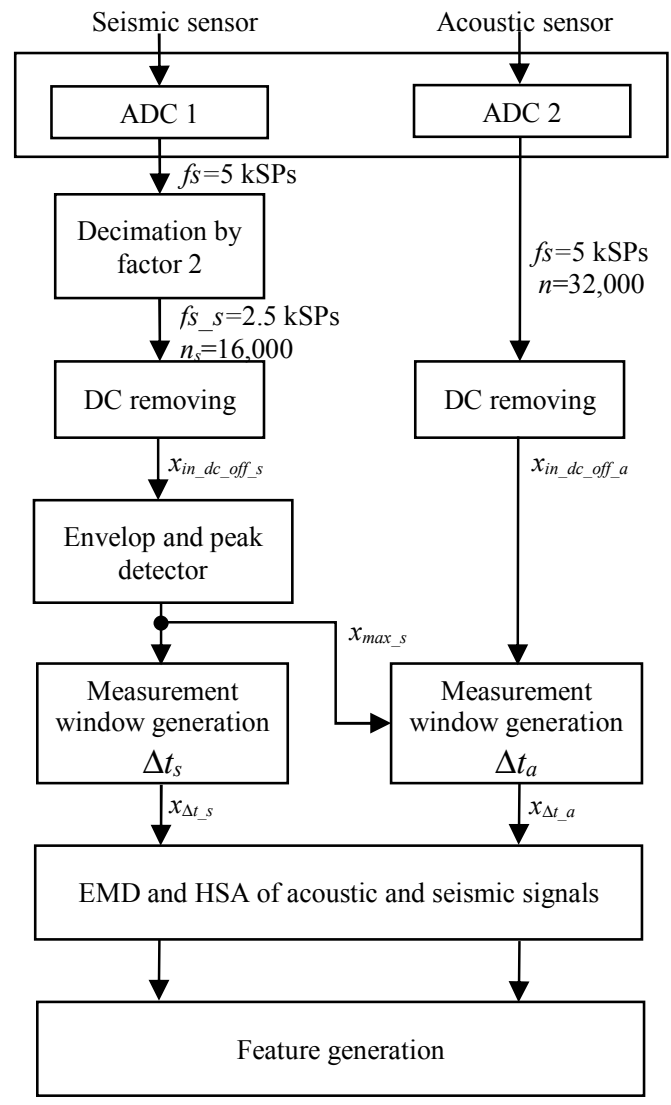

Figure 1. The flowchart of acoustic and seismic signal processing algorithms. 
The time series of seismic signal were transformed to vibration amplitude base on following formula [10]

$$
a=\frac{V_{o u t}}{K_{1}}-K_{0},
$$

where $a[\mathrm{~g}]$ denotes a vibration amplitude, $V_{\text {out }}[\mathrm{V}]$ denotes a level of output voltage, $K_{l}$ is the scale factor equal to $1.2 \mathrm{~V} / \mathrm{g}$ and $K_{0}[\mathrm{~g}]$ is a bias of the accelerometer. According to the assumption that the maximum amplitude of acoustic and seismic signal components are at the point when the vehicle is at the front of sensor position, only portion of the signal is necessary to use for further analysis. The distance between the vehicle and the sensor system placement was equal to $4.5 \mathrm{~m}$. The maximum amplitude of signal, as a parameter for defining of the measurement window $\Delta t$ was derived from the signal envelope. Due to the fact that the seismic signal is less sensitive to the environmental noise in comparison to acoustic one, envelop and peak detection algorithms were applied only to the seismic signal. The envelop detection algorithm is based on smoothed HT. It consists of the operation of filtration by using $5^{\text {th }}$ order low pass Butterworth filter with the cutoff frequency equal to 5 $\mathrm{Hz}$ and Hilbert transform of analytical signal. Obtained parameters describing the maximum amplitude $x_{\text {max } s}$ at the time $t_{\max }$ were also used for defining $\Delta t$ of the acoustic signal. Results of DC component removing; envelop and peak detection algorithms are shown in Fig. 2.

The length of the measurement window for the seismic signal is given by following formula

$$
\Delta t_{s}=t_{2}-t_{1}=t_{\max } \pm \Delta n \cdot T_{s_{-} s},
$$

where $\Delta n$ denotes a number of samples in time interval from $t_{\max }$ to $t_{l}$, from $t_{\max }$ to $t_{2}$, and $T_{s_{-} s}$ represents the sampling period of the seismic signal. Similarly, the length of the measurement window for acoustic signal is given by

$$
\Delta t_{a}=t_{2}-t_{1}=t_{\max } \pm \Delta n \cdot T_{s},
$$

where $T_{s}$ represents sampling period of the acoustic signal. The number of samples involved in the measurement window, results the time interval equal to $1 \mathrm{sec}(5,000$ samples for acoustic signal and 2,500 samples for seismic one). These samples were used as input signals for the time-frequency analysis by using HEDS. The HHT provides an alternative view of the time-frequency energy paradigm of data [8]. It provides better possibility to deal with the analysis of nonstationary and nonlinear signals in the timefrequency domain by finding instantaneous amplitude (4) and frequency (6) in comparison of traditional time-frequency signal analysis methods, like STFT. The time and frequency domain was divided into $128 \times 128$ bins. The resolution was set with respect to the character of seismic and acoustic signals generated by moving personal vehicles. With regards to the number of seismic signal samples and the sampling frequency of seismic signal, the resolution in time domain is equal to $7.81 \mathrm{~ms}$ and frequency one is equal to $9.84 \mathrm{~Hz}$. The time-frequency representation of the seismic signal by using HEDS is shown in Fig. 3.
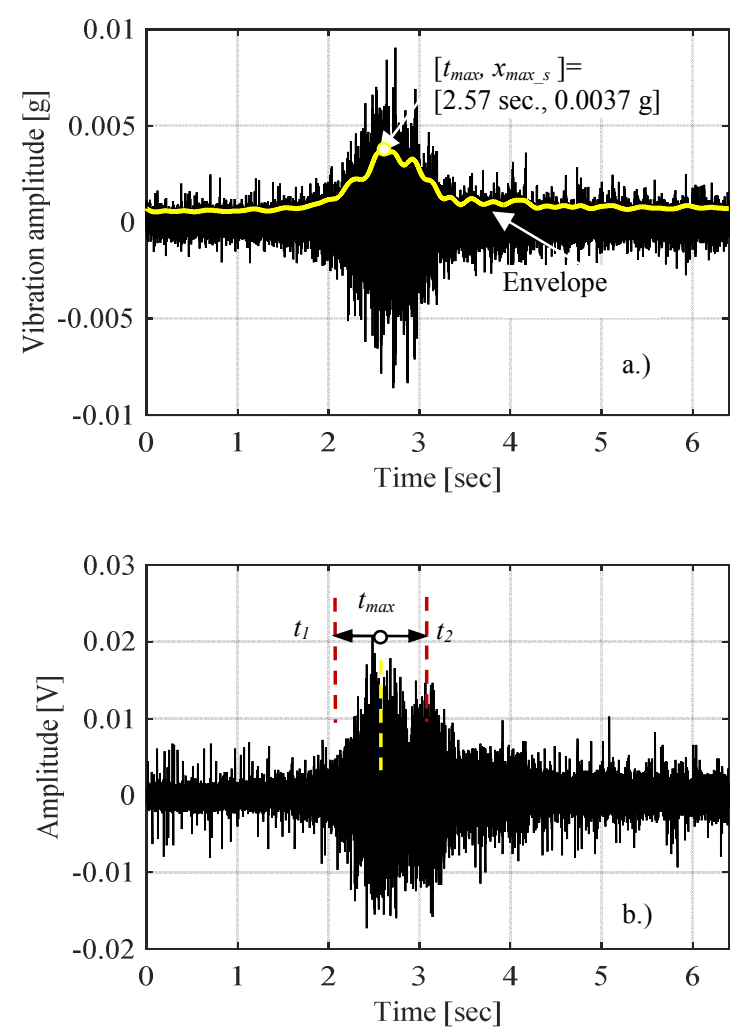

Figure 2. Time representation of seismic (a.) and acoustic (b.) signal generated by moving vehicle.

The event generated by the moving vehicle can be described by allocation of significant frequency components in the time-frequency space of HEDS. As an initial assumption for the feature generation, the number of significant frequency components $(p)$ was set to $2^{k}$. For our case, the parameter $k$ is equal to 3 which defines the size of the feature vector equal to $p=8$. The algorithm for detection of main frequency components is based on: 1.) detection of the frequency component $f_{l}$ with maximum energy, 2.) detection of the frequency component $f_{2}<f_{1}, 3$.) set $f_{2}$ as temporal significant frequency component, 4.) detection of next significant frequency component $f_{3}<f_{2}, 5$.) finish the detection process when the number of significant frequency components is equal to 8 .

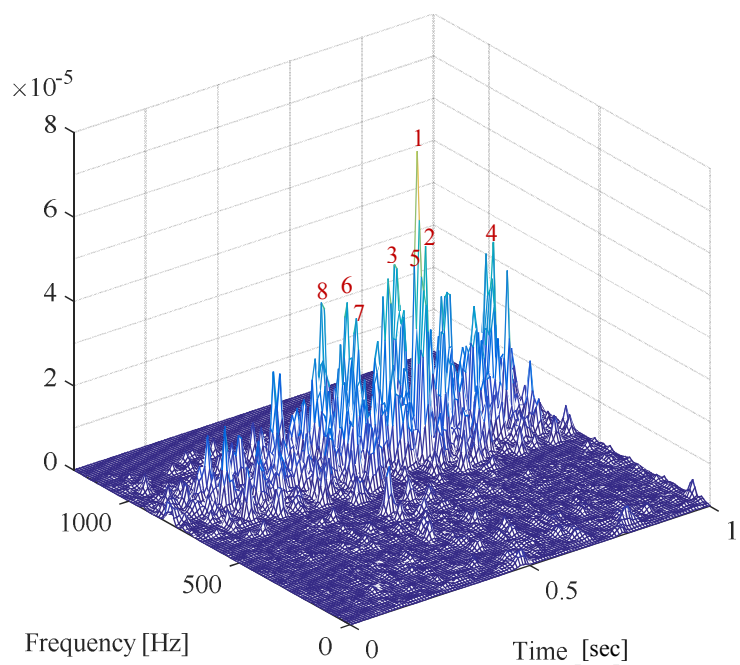

Figure 3. Hilbert energy density spectrum of seismic signal. 
The feature vector is defined by following expression

$$
F V=\left(f_{1}, f_{2}, \ldots, f_{p}\right),
$$

Due to the fact that the acoustic signal has for the same time interval higher number of samples than the seismic one, different resolution in frequency was achieved for given size of bins (128x128). The time resolution is equal to $7.81 \mathrm{~ms}$ and frequency one is equal to $19.68 \mathrm{~Hz}$. The representation of HEDS for acoustic signal is shown in Fig. 4.

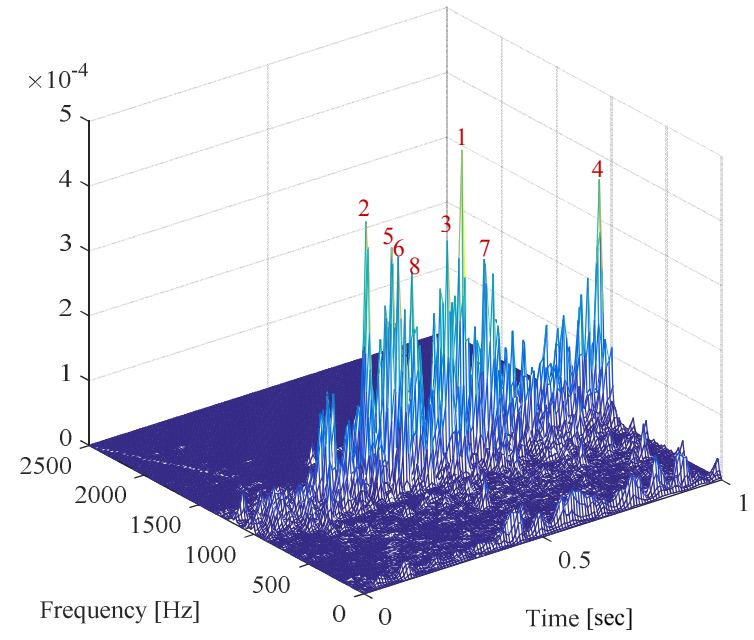

Figure 4. Hilbert energy density spectrum of acoustic signal.

The results, shown in Table I. are arranged from frequency component with the highest energy $\left(f_{l}\right)$ to the lowest one $\left(f_{8}\right)$. From the results imply that detected significant frequency components of acoustic and seismic signals occupied not same frequency bands. Detected significant frequency components of the seismic signal are located in the frequency band from $748 \mathrm{~Hz}$ to $885 \mathrm{~Hz}$, while the acoustic ones are located in the frequency band from 1108 to $1157 \mathrm{~Hz}$. There are several acoustic and seismic frequency components (highlighted fields in Table I.) which have about equally time spots of occurrence in the timefrequency representation of HEDS.

TABLE I. SELECTED SIGNIFICANT FREQUENCY COMPOONENTS OF SEISMIC AND ACOUSTIC SIGNALS

\begin{tabular}{|c|c|c|c|c|c|}
\hline \multicolumn{3}{|c|}{$\begin{array}{c}\text { Parameters of seismic } \\
\text { signal components }\end{array}$} & \multicolumn{3}{|c|}{$\begin{array}{c}\text { Parameters of acoustic } \\
\text { signal components }\end{array}$} \\
\hline time [ms] & $\mathbf{f}[\mathbf{H z}]$ & $\mathbf{A}\left[\mathbf{1 0}^{-5}\right]$ & time [ms] & $\mathbf{f}[\mathbf{H z}]$ & $\mathbf{A}\left[\mathbf{1 0}^{-5}\right]$ \\
\hline 0.64 & 748 & 7.203 & 0.61 & 1108 & 47.4 \\
\hline 0.66 & 748 & 4.88 & 0.34 & 1108 & 41.1 \\
\hline 0.58 & 777 & 4.2 & 0.54 & 1010 & 36.4 \\
\hline 0.905 & 826 & 4.084 & 0.99 & 1088 & 35.62 \\
\hline 0.66 & 866 & 4.039 & 0.4 & 1020 & 35.44 \\
\hline 0.57 & 787.4 & 3.83 & 0.43 & 1157 & 35.17 \\
\hline 0.51 & 846 & 3.7 & 0.66 & 1059 & 30.88 \\
\hline 0.46 & 885 & 3.6 & 0.48 & 1127 & 30.25 \\
\hline
\end{tabular}

The most of these components are located in time interval within $\pm 100 \mathrm{~ms}$ from the center of measurement window $\left(t_{\max }\right)$. There is one exception, when only $4^{\text {th }}$ frequency component is located at the end of the time interval of measurement window.

\section{CONCLUSIONS}

Due to the nonlinear and nonstationary character of seismic and acoustic signals generated by moving vehicles, the HHT provides the appropriate way for the signal analysis in the time-frequency domain. From the time representation of seismic and acoustic signals implies that even signals have a noisy character, it is possible to generate the measurement window base on maximum amplitude of seismic signal envelop. Results (shown in Table I.) point to the fact, that time interval equal to $200 \mathrm{~ms}$ is sufficient for detection significant frequency components, which can be used for feature generation. For this time interval, the number of samples of acoustic signal is equal to 1,000 and for seismic one is equal to 500. Proposed algorithms provide the possibility to reduce the number of samples required for feature generation based on the HEDS.

Future work will be focused on the statistical confirmation of analysis outcomes, in terms of the generation of optimal size of feature vector and optimization of algorithms for their implementation in sensor system based on digital signal processor.

\section{REFERENCES}

[1] H. W. Zhou, Practical seismic data analysis, Cambridge University Press, 2014, pp. 66-78.

[2] S. Theodoridis, and K. Koutroumbas, Pattern Recognition, Elsevier, 2009, pp. 363-366.

[3] S. Qian, Introduction to Time-Frequency and Wavelet transforms, Prentice Hall PTR, 2002, pp. 51-52.

[4] N. E. Huang, and S. S. P. Shen, Hilbert-Huang transform and its applications, World Scientfic Publishing, 2005, pp. 1-24.

[5] R. Berešík, "Hilbert-Huang transform and its application in seismic signal processing," in 2016 International Conference on New Trends in Signal Processing (NTSP), October 2016, pp. 1-6.

[6] Z. Wu, and N. E. Huang, "Ensemble empirical mode decomposition: A noise-assisted data analysis method," in Advances in Adaptive Data Analysis, vol. 1, no.1, 2009, pp. $1-41$.

[7] M. E. Torres, M. A. Colominas, G. S. Schlotthauer, and P. Flandrin, "A Complete Ensemble Empirical Mode Decomposition with Adaptive Noise," in 2011 IEEE International Conferecne on Acoustic, Speech and Signal Processign (ICASSP), May 2011, pp. 4144-4147.

[8] N. E. Huang and Z. Wu, "A Review on Hilbert-Huang Transform: Method and its Applications to Geophysical Studies," in Reviews of Geophysics, 46, RG2006/2008, pp. 123.

[9] N. E. Huang, X. Chen, M. T. Lo, and Z. Wu, "On Hilbert spectral representation: A true time-frequency representation for nonlinear and nonstationary data," in Advances in Adaptive Data Analysis, vol. 3, no. 1 and 2, 2011, pp. 63-93.

[10] Colibrys, MEMS Capacitive AccelerometersSF1500S.A/SF1500SN.A, 30S.SF1500A.E.09.10, Colybris, 2010.

[11] Analog Devices, Omnidirectional Microphone with Bottom Port and Analog Output, ADMP404, Analog Devices, 2012. 